\title{
Transplantation of endothelial progenitor cells transfected with VEGF165 to restore erectile function in diabetic rats
}

\author{
Xin Gou, Wei-Yang He, Ming-Zhao Xiao, Ming Qiu, Ming Wang, Yuan-Zhong Deng, Chao-Dong Liu, \\ Zao-Bing Tang, Jie Li and Yong Chen
}

The present study investigated the effect of transplanting endothelial progenitor cells (EPCs) transfected with the vascular endothelial growth factor gene (VEGF165) into the corpora cavernosa of rats with diabetic erectile dysfunction (ED). A rat model of diabetic ED was constructed via intraperitoneal injection of streptozotocin. After streptozotocin treatment, pre-treated EPCs from each of three groups of rats were transplanted into their corpora cavernosa. Our results, following intracavernosal pressure (ICP) monitoring, showed that ICP increased significantly among rats in the trial group when compared to the results from rats in the blank-plasmid and control groups during basal conditions and electrical stimulation $(\boldsymbol{P}<0.01$ for both comparisons). Histological examination revealed extensive neovascularisation in the corpora cavernosa of rats in the trial group. Fluorescence microscopy indicated that many of the transplanted EPCs in the trial group survived, differentiated into endothelial cells and integrated into the sites of neovascularisation. Based on the results of this study, we conclude that transplantation of VEGF165-transfected EPCs into the corpora cavernosa of rats with diabetic ED restores erectile function.

Asian Journal of Andrology (2011) 13, 332-338; doi:10.1038/aja.2010.116; published online 29 November 2010

Keywords: cell transplantation; diabetes mellitus; endothelial progenitor cells; erectile dysfunction; gene expression; vascular endothelial growth factor

\section{INTRODUCTION}

Penile erection is a complicated physiological event that involves many psychological, neural, vascular and endocrine factors. Erectile dysfunction (ED) is one of the major complications of diabetes mellitus. ${ }^{1}$ Burke et al. ${ }^{2}$ studied 2115 white male diabetic patients, aged 40-79 years, and reported that approximately $50 \%$ had ED. Recent studies 3 have revealed that direct injection of the vascular endothelial growth factor (VEGF) gene or protein into the corpora cavernosa restored erectile function in rabbits with hypercholesterolemia-induced ED and rats with traumatic arteriogenic ED. However, the viral vectors that are used for transduction may undergo recombination with endogenous viruses and may also cause cancer and elicit immune responses, restricting the extensive application of this method. In recent years, the use of ex vivo-transfected stem cells as vectors for gene therapy has largely solved this problem. Bivalacqua et al. ${ }^{5}$ transplanted ex vivo endothelial nitric oxide synthase gene-modified mesenchymal stem cells into the corpora cavernosa of geriatric rats and was able to restore their erectile function. However, there are few reports regarding the use of ex vivo VEGF gene-modified endothelial progenitor cells (EPCs) to treat diabetic ED in rats.

Our hypothesis was that the transplantation of ex vivo VEGF genemodified EPCs into the corpora cavernosa of rats with diabetic ED would restore their erectile function by facilitating the expression of $V E G F$ and promoting neovascularisation.

\section{MATERIALS AND METHODS}

Materials

M199 medium, pcDNA3.1(+), pCR2.1-TOPO-VEGF165 vector and Lipofectamine 2000 were obtained from Invitrogen (Carlsbad, CA, USA); Ficoll was purchased from TBD (Shanghai, China); foetal bovine serum was obtained from Gibco (Shanghai, China); and VEGF was purchased from R\&D (Atlantic, FL, USA). Rabbit anti-rat CD34 and CD133 antibodies were purchased from Beijing Boaosen (Beijing, China); rabbit anti-rat KDR antibody was obtained from Santa Cruz (Santa Cruz, CA, USA); the VEGF ELISA Kit was purchased from Xinbosheng (Beijing, China); streptozotocin, fibronectin and apomorphine were purchased from Sigma (St Louis, MO, USA); the RT-PCR Kit was obtained from TaKaRa (Dalian, China); 2-(4-amidinophenyl)-1H-indole-6-carboxamidine (DAPI) was purchased from Biyuntian (Shanghai, China); and the DNA Extraction Kit was obtained from the Omega Company (Houston, TX, USA). The fluorescence microscope was supplied by Olympus (Tokyo, Japan), and the instantaneous bio-signal recorder was supplied by Chengdu Taimeng (Chengdu, China). 
Animals were supplied by the Laboratory Animal Center of Chongqing Medical University (Chongqing, China). All animal protocols were approved by Institutional Animal Care and Use Committee of Chongqing Medical University.

\section{Methods}

Isolation of EPCs. Femurs and tibias were removed from 2-week-old Sprague-Dawley rats under sterile conditions. Their bone marrow cavities were rinsed with $5-10 \mathrm{ml}$ of $0.01 \mathrm{~mol} \mathrm{l}^{-1}$ phosphate-buffered saline, precooled to $4{ }^{\circ} \mathrm{C}$. The rinsing solution was then mixed thoroughly and transferred into Ficoll-supplemented centrifuge tubes followed by centrifugation at $358 \mathrm{~g}$ and $20{ }^{\circ} \mathrm{C}$ for $20 \mathrm{~min}^{6-8}$ The mononuclear cells remaining in the intermediate layer were then carefully aspirated and transferred to new centrifuge tubes. Mononuclear cells were rinsed with phosphate-buffered saline and precipitated by centrifugation twice, and then resuspended in M199 medium and distributed into $100 \mathrm{ml}$ culture flasks and 12-well plates at a concentration of $1 \times 10^{6}$ cells $\mathrm{ml}^{-1}$. Two hours before cell seeding, cover slips were placed at the bottom of the 12-well plates and the flasks and plates were coated with fibronectin at a density of $5 \mu \mathrm{g} \mathrm{cm}{ }^{-2}$. Seeded cells were cultured at $37^{\circ} \mathrm{C}$ in a humidified incubator containing $5 \% \mathrm{CO}_{2}$. Immunohistochemical analyses were conducted to characterize the expression of CD34, KDR and CD133 on adherent cells.

Construction of the pcDNA3.1(+)/NEGF165 expression vector ${ }^{9,10}$. Plasmid pCR2.1-TOPO-VEGF165 was restriction digested with HindIII and XhoI, and the resultant 580-bp (VEGF165) fragment was purified. The pcDNA3.1(+) plasmid was also double-digested with HindIII and XhoI. The VEGF165 fragment was then ligated into the digested pcDNA3.1(+) fragment to construct the expression vector pcDNA3.1 $(+) / V E G F 165$. The recombinant vectors were transformed into the competent Escherichia coli strain $\mathrm{DH} 5 \alpha$ for amplification. Next, the plasmids were extracted and characterized by sequencing, restriction analysis and PCR.

EPC transfection and the detection of VEGF165 gene or protein levels in EPCs. Transfection was conducted according to the instructions of the Lipofectamine 2000 Mammalian Transfection Kit. Briefly, once EPCs reached 50\% confluence and were refreshed with serum-free M199 medium, they were transfected with either the expression vector pcDNA3.1(+)/VEGF165 or the unmodified plasmid pcDNA3.1 $(+)$ (the plasmid/liposome ratio was 1:3) in 24-well plates. The serumcontaining medium was refreshed $24 \mathrm{~h}$ after transfection. The VEGF165 gene-modified EPCs were then selected using $600 \mu \mathrm{g} \mathrm{ml} \mathrm{gl}^{-1}$ G418. Selected EPCs were harvested and transferred into M199 medium containing $20 \%$ foetal bovine serum. The supernatants were harvested at days $1,3,5,7,9,11$ and 13 post-transfection. Following harvest, samples were centrifuged and then stored at $-70{ }^{\circ} \mathrm{C}$. A human VEGF ELISA Kit was used to detect the concentration of VEGF165 protein in the supernatants in accordance with the manufacturer's instructions. The total RNA content of the harvested EPCs was extracted using the conventional TRIzol method, and RT-PCR was conducted to detect the expression of the VEGF165 gene. Primers were designed using the guidance of the NIH GenBank and their sequences are listed as below:

1. VEGF165 (GenBank, AB021221) primers: upstream primer 5' AAGCTTGCCACCATGAACTTTCTGCTGT-3'; downstream primer 5'-CTCGAGTCACCGCCTCGGCTTGTC-3';

2. (2) Internal control $\beta$-actin primers: upstream primer $5^{\prime}$ CCGTAAAGACCTCTATGCCAAC-3'; downstream primer 5' ACTCATCGTACTCCTGCTTGCT-3'.
Creation of a rat model of diabetic ED. A rat model of diabetic ED was created as described. ${ }^{11,12}$ A total of 60 8-week-old male SpragueDawley rats, weighing 230-260 g, underwent intraperitoneal injection of $1 \%$ streptozotocin solution $\left(65 \mathrm{mg} \mathrm{kg}^{-1}\right)$ in their lower left quadrants after a 12-h fast during which they were only allowed access to water. A total of $72 \mathrm{~h}$ after injection, blood was aspirated from the tail vein of each rat for random blood glucose measurements. Rats with blood glucose concentrations higher than $16.7 \mathrm{mmol} \mathrm{l}^{-1}$ were diagnosed with diabetes. Rats that met this criterion and continued feeding for 14 weeks had another random blood glucose measurement taken from their tail veins. Later, apomorphine solution was prepared and injected into the soft skin on the necks of the rats at a dose of $100 \mu \mathrm{g} \mathrm{kg}^{-1}$. The duration of erections during a $30-\mathrm{min}$ period were recorded. Rats that had a blood glucose concentration greater than $7.2 \mathrm{mmoll}^{-1}$ and a negative response to apomorphine induction were considered to be appropriate models of diabetic ED. In total, 35 rat models of diabetic ED were successfully generated.

EPC transplantation. Rats were randomized into three treatment groups as follows: (i) trial group: EPCs transfected with pcDNA3.1(+)/VEGF165 were transplanted into 13 rats; (ii) blankplasmid group: EPCs transfected with blank pcDNA3.1(+) were transplanted into 12 rats; and (iii) control group: EPCs that had not been transfected were transplanted into 10 rats. Before transplantation, the EPCs of each group were labelled with DAPI (for blue fluorescence). The EPCs $\left(0.2 \mathrm{ml}\right.$ at a concentration of $1 \times 10^{7}$ cells $\left.\mathrm{ml}^{-1}\right)$ were then injected into the base of the penis.

Intracavernosal pressure (ICP) measurement. ICP was measured as described. ${ }^{13-17}$ Twenty-one days after EPC transplantation, rats were anesthetized with an intraperitoneal injection of $3 \%$ pentobarbital sodium and fixed in the supine position. We then performed a midline incision of the lower abdomen. The pelvic ganglia and cavernous nerves were exposed using a surgical microscope ( $\times 10$ magnification). A bipolar electrode connected to an electrical pulse stimulator was placed onto the cavernous nerves. Next, an additional oblique incision was performed to allow dissection and exposure of the corpora penis. A 23-gauge butterfly needle connected to a pressure recorder (to allow recording of the ICP values) was then inserted into the crus penis. Before needle insertion, sterilized heparin (100 U heparin/ml) was aspirated into the needle to prevent occlusion due to blood coagulation. The cavernous nerves were then stimulated by sustained electrical pulses with a pulse width of $0.5 \mathrm{~ms}$, a frequency of $16 \mathrm{~Hz}$ and an intensity of $10 \mathrm{~V}$. A single stimulation lasted for a total of $60 \mathrm{~s}$, and the interval between two single stimulations was 2-3 min. After an additional intraperitoneal injection of pentobarbital, the ICP values for each stimulation were recorded. After an additional intraperitoneal injection of pentobarbital, the ICP values for each stimulation were recorded.

Detection of in vivo VEGF165 gene and protein levels via ELISA and RTPCR. At days 1, 4, 7, 14 and 21 post-EPC transplantation, the peripheral blood of each rat was sampled and centrifuged. The supernatants were then stored at $-70{ }^{\circ} \mathrm{C}$. A VEGF ELISA Kit was used to measure the concentration of VEGF165 protein in accordance with the manufacturer's instructions. A total of 21 days after EPC transplantation, rats were killed by administration of an overdose of $3 \%$ pentobarbital sodium. The total RNA content of the corpora cavernosa of the rats was extracted by the conventional TRIzol method, and RT-PCR was performed to detect the expression of the VEGF165 gene. 
Visualisation of blood vessels in the corpus cavernosum via immunohistochemistry. The corpora cavernosa of each rat was fixed with $10 \%$ neutral formalin buffer for $24 \mathrm{~h}$, rinsed with phosphate-buffered saline and stored in $75 \%$ ethanol. The fixed sections were then stained with haematoxylin and eosin (HE). Rabbit anti-CD34 antibody was used for subsequent immunohistochemical staining, in accordance with the manufacturer's specifications, in order to label the blood vessels in the corpora cavernosa. Five randomly selected microscopic visual fields for each stained section were examined. All CD34-positive structures were determined to be capillary vessels and based on the CD34 staining pattern, the number of capillary vessels was determined and the microvessel density was calculated. All morphometric evaluations were performed by two examiners who were blinded to the treatments the rats had received.

Examination of the corpus cavernosum via histological fluorescence. Tissue sections of the corpora cavernosa from the rats included in the study were observed by fluorescence microscopy. The DAPIlabelled EPCs produced a blue signal under the fluorescence microscope, and the degree and intensity of blue fluorescence reflected the cellular content and degree of proliferation of EPCs in the corpora cavernosa of the rats.

Statistical methods. The SPSS 13.0 statistical software (SPSS Inc., Chicago, IL, USA) was used for data analysis. We performed variance analysis on our quantitative data using an $\alpha$ level of 0.05 . $P<0.05$ was considered statistically significant.

\section{RESULTS}

\section{Isolation of EPCs}

The isolated EPCs were initially small and round. After $24 \mathrm{~h}$, a few cells began to adhere and grew larger. After 4 days in culture, adherent EPCs began to proliferate. The EPC colonies included round cells in the middle and fusiform cells in the periphery, similar to the morphology exhibited by these cells in embryonic blood islands. As shown in Figure 1, EPCs exhibited endothelium-specific cord-like structures and reached approximate confluence after 12 days in culture. After they were transferred into a new culture medium, the EPCs continued to grow, and the fusiform EPCs arranged themselves in an end-to-end conformation that resembled a capillary. Figure 2 shows that CD34, KDR and CD133 were all expressed by adherent EPCs (based on our immunohistochemical assays). The expression of these factors is characteristic of that by EPCs. ${ }^{18}$

\section{Construction of the pcDNA3.1-VEGF165 expression vector}

The recombinant plasmid DNA fragments were amplified in E. coli, extracted and double-digested by HindIII and XhoI. The resulting DNA fragments were then separated by gel electrophoresis. Gel image analysis revealed the presence of a bright band between 500 and $600 \mathrm{bp}$ in length, which was consistent with VEGF165 cDNA (Figure 3a). PCR was conducted using the recombinant plasmid extract as a template. Gel image analysis of the PCR products showed a bright bands of about $576 \mathrm{bp}$ in length (Figure $3 \mathrm{~b}$ ) and the results of sequencing (Shanghai Yingjun, Shanghai, China) showed that this band's sequence was consistent with the GenBank VEGF165 sequence.

\section{VEGF165 gene and protein levels in EPCs}

The results of this study indicate that EPCs transfected with a recombinant plasmid containing the VEGF165 gene expressed significantly more VEGF165 protein than those that were transfected with a blank plasmid or were not transfected at all (Figure 4a). Gel image analysis of the RT-PCR products from these three groups showed bands of varying brightness that were between 500 and 700 bp in length (Figure $4 \mathrm{~b}$ ). The EPCs that had been transfected with blank plasmids or that had not been transfected exhibited weaker bands than EPCs transfected with recombinant plasmid because the EPCs from the former two groups secreted only small amounts of VEGF165.

\section{ICP measurements}

The mean basal ICP values of the trial group, blank-plasmid group and control group were 9.32 $\pm 3.23,6.47 \pm 1.56$ and $6.14 \pm 2.16 \mathrm{cmH}_{2} \mathrm{O}$, respectively. The mean basal ICP of the trial group was notably higher than those of the other groups $(P<0.01)$. After electrical stimulation, the ICP values of the trial group, blank-plasmid group and control group were $56.22 \pm 9.56,20.46 \pm 4.67$ and $20.24 \pm 5.48 \mathrm{cmH}_{2} \mathrm{O}$, respectively. This result indicated that the mean electrical stimulusinduced ICP value of the rats in the trial group was also notably higher than those of the other groups after VEGF165 gene-modified EPC transplantation $(P<0.01)$ (Figure 5).

\section{In vivo VEGF165 gene and protein levels}

The results in Figure 6a show that the trial group had remarkably higher in vivo VEGF165 levels than the blank-plasmid group and control group $(P<0.05)$. The VEGF expression peaked at 7 days after transplantation and then gradually declined. The results of the RTPCR analysis indicated that the VEGF165 gene was abundantly expressed in the corpora cavernosa of the trial group 21 days after
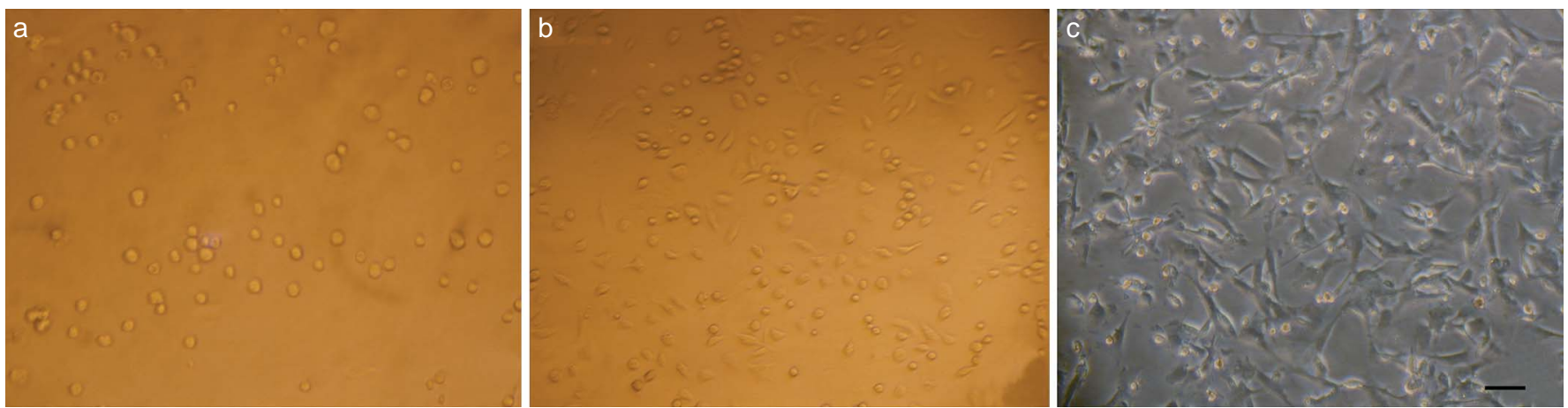

Figure 1 Microscopic images of EPCs ( $\times 40$ magnification). (a) A total of $24 \mathrm{~h}$ after initial isolation, EPCs were small and round, with only a few adherent cells and larger cells. (b) After 4 days of culture, EPCs demonstrated colony formation. (c) After 12 days of culture, fusiform EPCs were arranged in an end-to-end conformation, resembling the morphology of a capillary. Bar $=20 \mu \mathrm{m}$. EPC, endothelial progenitor cell. 

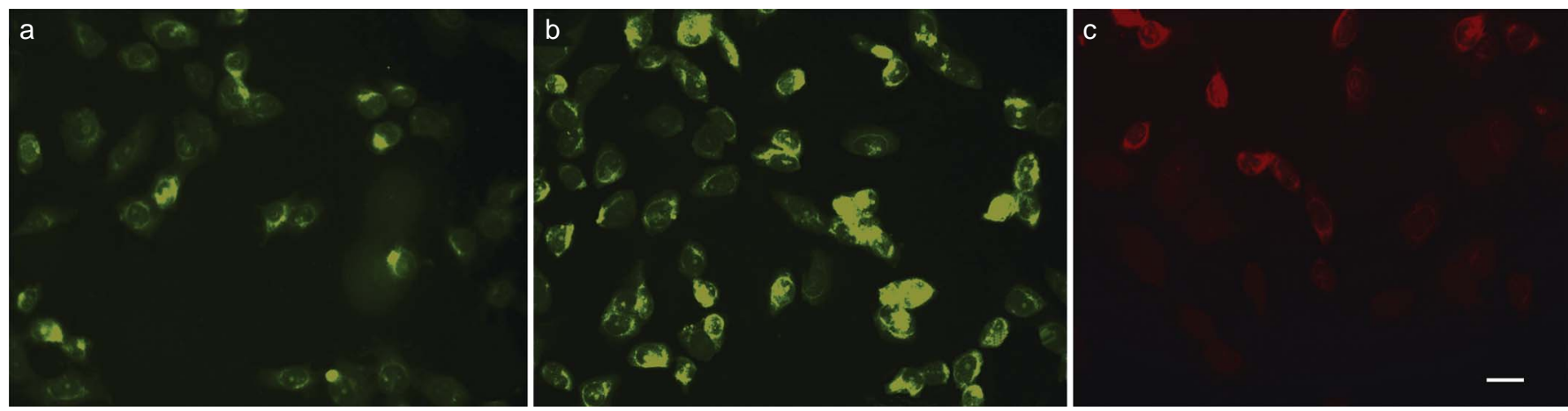

Figure 2 Fluorescence microscopy images of EPCs (×200 magnification). (a) CD34-positive; (b) KDR-positive; (c) CD133-positive cells. Bar=100 $\mu$ m. EPC, endothelial progenitor cell.
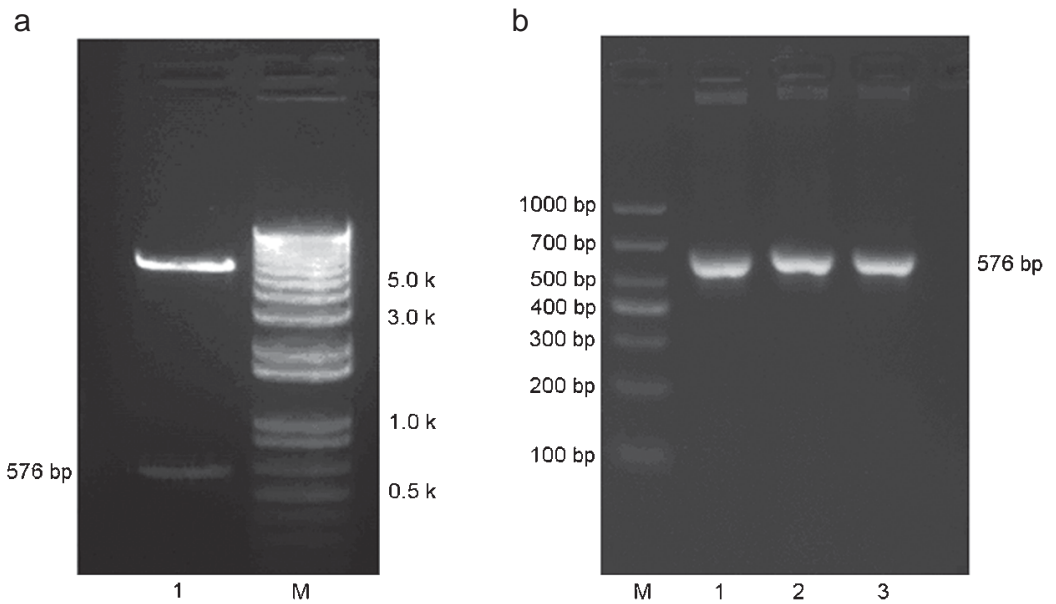

Figure 3 Construction of the pcDNA3.1-VEGF165 expression vector. (a) Characterisation of recombinant pcDNA3.1-VEGF165 via enzyme digestion. Lane 1: The DNA fragments that were present after double digestion by Hindlll and Xhol, including a bright band that was between 500 and 600 bp in length; M: DNA marker. (b) Characterisation of recombinant pcDNA3.1-VEGF165 via PCR. Lanes 1-3: PCR-amplified products of the VEGF165 insert that were between 500 and 700 bp in length; M: DNA marker. VEGF, vascular endothelial growth factor.
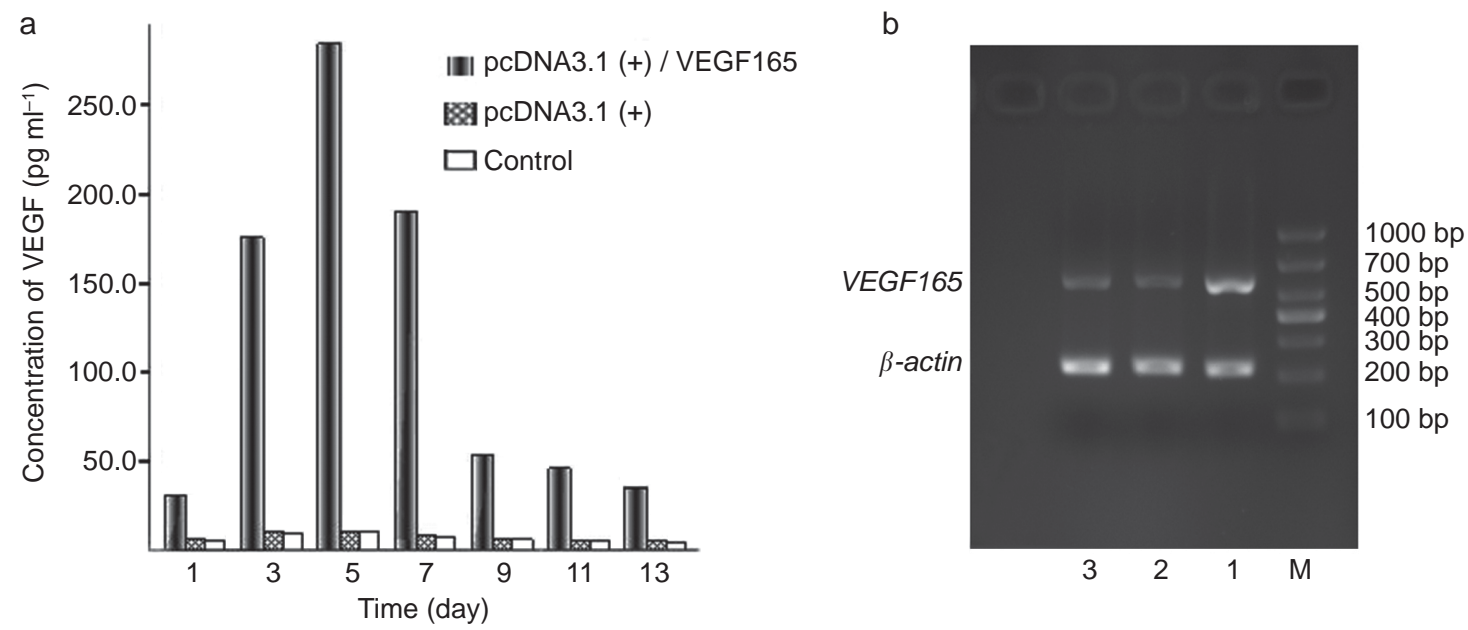

Figure 4 VEGF165 gene and protein levels in EPCs. (a) The levels of VEGF165 protein secreted by EPCs, as assessed by ELISA. (b) Determination of the expression levels of the VEGF165 gene in EPCs via RT-PCR. The lane on the left is the basal ICP. Lane 1: EPCs that were transfected with pcDNA3.1(+)/VEGF165 recombinant plasmid. Lane 2: EPCs that were transfected with blank pcDNA3.1(+) plasmid. Lane 3: EPCs that were not transfected. M: DNA marker; Lanes 1-3 exhibited bands between 500 and 700 bp in length. The band in Lane 1 was the brightest of all three bands. EPC, endothelial progenitor cell; ICP, intracavernosal pressure; VEGF, vascular endothelial growth factor. 
a

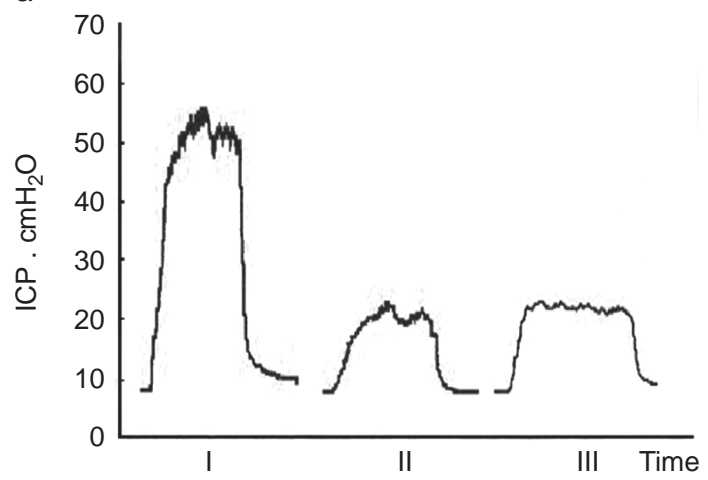

b

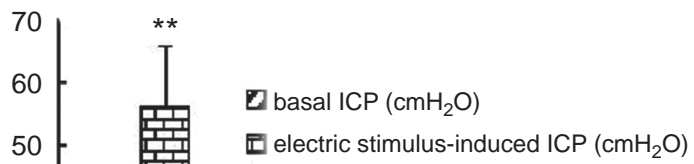

Figure 5 ICP measurements. (a) Changes in the ICP of rats after electrical stimulus. (b) The basal ICP (refer to value of ICP after transplanting EPCS transfected with VEGF165, but before electrical stimulus) and electrical stimulus-induced ICP values of rats with diabetic ED. I, trial group; II, blank-plasmid group; III, control group. ${ }^{*} P<0.01,{ }^{*} P<0.01$, compared with II and III. ED, erectile dysfunction; EPC, endothelial progenitor cell; ICP, intracavernosal pressure (P,0.01); VEGF, vascular endothelial growth factor.

EPC transplantation and that there were lower levels of expression in the blank-plasmid group and the control group at this time point (Figure 6b).

\section{Neovascularisation in the corpus cavernosum}

The results of HE staining (Figure 7a) and CD34 vascular staining (Figure $7 \mathrm{~b}$ ) in the corpora cavernosa revealed numerous sites of neovascularisation in the rats in the trial group but fewer sites in the blank-plasmid group and the control group rats. The number of capillaries in the corpora cavernosa of rats in the trial group $\left(110.2 \pm 14.5 \mathrm{~mm}^{-2}\right)$ was significantly higher than that in blank-plasmid group $\left(62.0 \pm 9.8 \mathrm{~mm}^{-2}\right)$ and control group $\left(50.4 \pm 13.1 \mathrm{~mm}^{-2}\right)(P<0.01)$.

\section{Examination via fluorescence microscopy of the corpus cavernosum after EPC transplantation}

EPCs exhibited blue fluorescence under the fluorescence microscopy (Figure 8a) because the DAPI staining of the nuclei of the original EPCs was transferred into daughter cells via cell division. Thus, the fluorescence granules reflected the numbers of EPCs and endothelial cells (ECs) that were present in the corpora cavernosa of rats receiving transplants. Three weeks after EPC transplantation, the number of fluorescence granules in the trial group (219.6 $\pm 21.5 /$ highpower field) was significantly higher than that in blank-plasmid group (106.8 $\pm 11.9 /$ highpower field) and control group (119.8 $\pm 10.1 /$ highpower field) $(P<0.01)$ (Figure $8 \mathrm{~b})$.

\section{DISCUSSION}

EPCs are a type of precursor cells that differentiate into vascular ECs. EPCs induce ECs to repair blood vessels, create collateral circulation systems and restore the blood supply to ischemic tissues or organs. ${ }^{19,20}$ EPCs have exhibited certain characteristics that make EPC transplantation a promising potential treatment strategy for ischemic cardiomyopathy. ${ }^{21}$ Foresta et al. ${ }^{22}$ reported that there were remarkably fewer EPCs in the peripheral blood of patients with ED than in the blood of normal controls. They believed that EPC concentration in the peripheral blood could be a marker of ED, indicating that EPCs are involved in the pathogenesis of ED.

$V E G F$ is a specific type of cytokine that plays an important role in angiogenesis. Specifically, it binds to particular receptors on ECs to
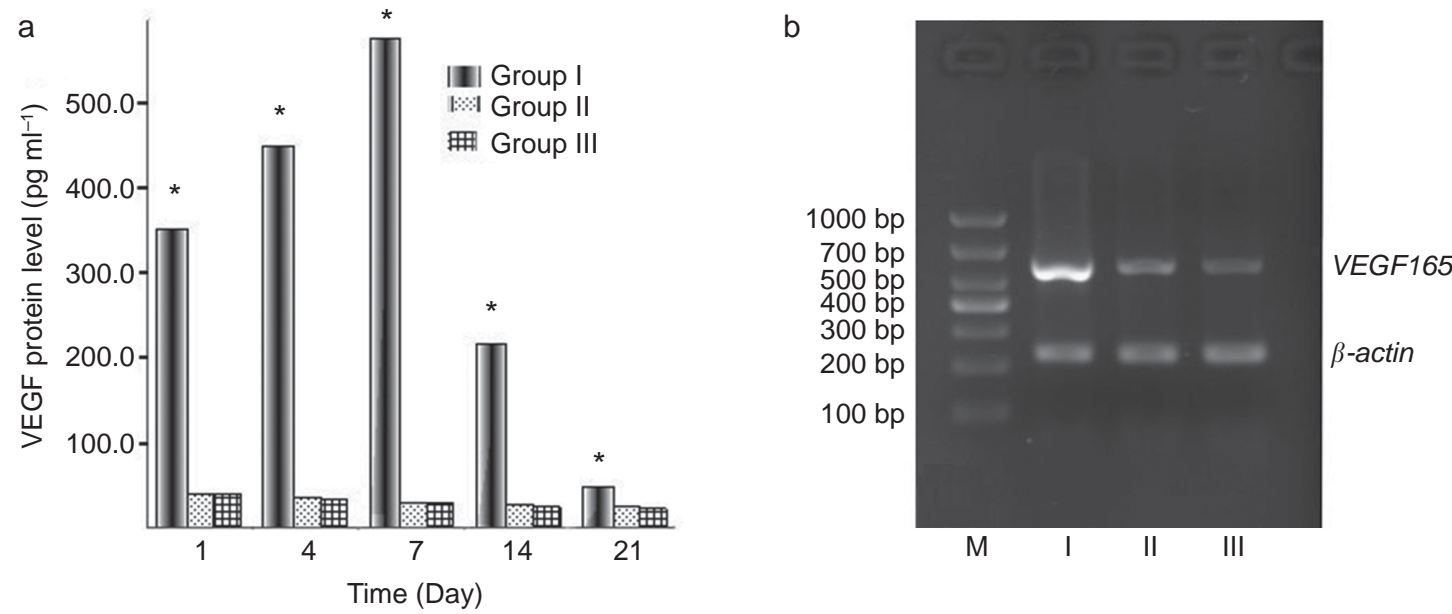

Figure 6 In vivo VEGF165 gene and protein levels. (a) VEGF165 protein levels in the peripheral blood, as assessed by ELISA. (b) VEGF165 gene expression in the penis 21 days after EPC transplantation as assessed by RT-PCR. Bands from the trial group (I), blank-plasmid group (II) and control group (III) all appeared to be between 500 and $700 \mathrm{bp}$ in length, but the band that belonged to the trial group was brighter than those of the other groups. M, DNA marker. * $P<0.05$, compared with blank-plasmid group and control group. EPC, endothelial progenitor cell; VEGF, vascular endothelial growth factor. 


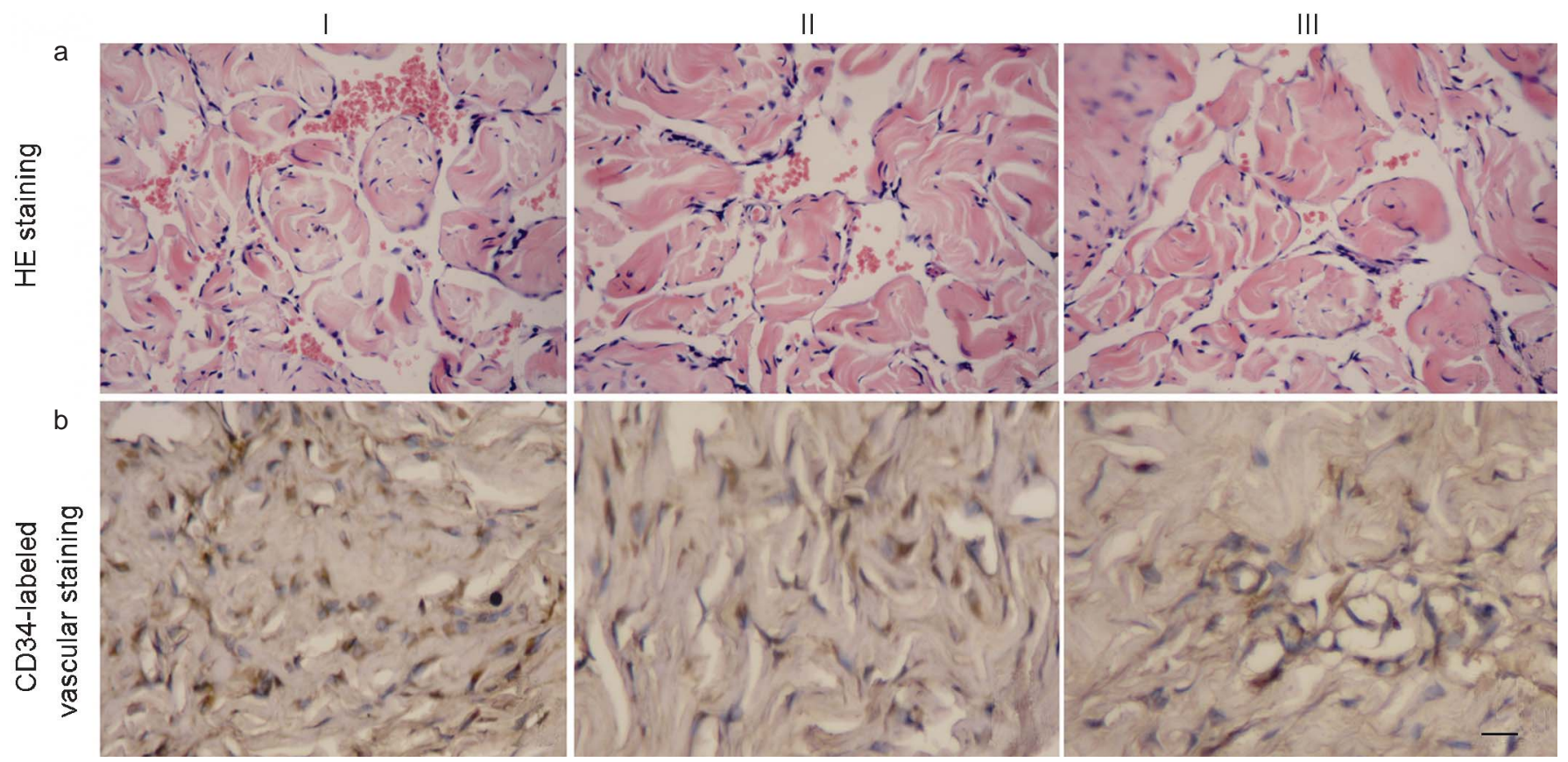

Figure 7 The results of HE staining and CD34 vascular staining in the corpora cavernosa. (a) Microscopic image of the corpus cavernosum after HE staining $(\times 200$ magnification). The number of capillaries in the corpora cavernosa of rats in the trial group $\left(110.2 \pm 14.5 \mathrm{~mm}^{-2}\right)$ was significantly higher than that in blank-plasmid group $\left(62.0 \pm 9.8 \mathrm{~mm}^{-2}\right)$ and control group $\left(50.4 \pm 13.1 \mathrm{~mm}^{-2}\right)(P<0.01)$. (b) Microscopic image of the corpus cavernosum after CD34-labelled vascular staining. The vessels in the penile tissues of the trial group (I), blank-plasmid group (II) and control group (III) were dyed yellow-brown. There are obviously more vessels present in the trial group than in the blank plasmid or control groups. Bar=20 $\mu \mathrm{m}$. HE, haematoxylin and eosin.
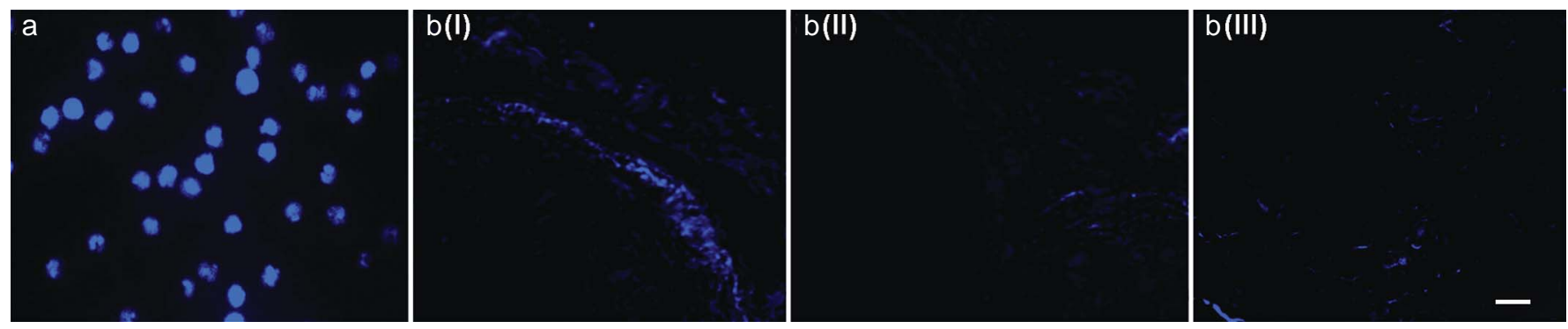

Figure 8 Examination via fluorescence microscopy of the corpus cavernosum after EPC transplantation. (a) DAPI-labelled EPCs ( $\times 200$ magnification). The nuclei of EPCs were labelled by DAPI and exhibited blue fluorescence when examined with a fluorescence microscope. (b) An image of the corpus cavernosum ( $\times 200$ magnification) obtained via fluorescence microscopy. The DAPI-labelled EPCs were transplanted into the corpora cavernosa and then proliferated, differentiated and incorporated into sites of neovascularisation that exhibited blue fluorescence when examined with a fluorescence microscope. I, trial group; II, blank-plasmid group; III, control group. Bar=80 $\mu \mathrm{m}$. DAPI, 2-(4-amidinophenyl)-1H-indole-6-carboxamidine; EPC, endothelial progenitor cell.

facilitate angiogenesis, enhance vascular permeability and maintain vascular function. ${ }^{23,24}$ In various types of ED, the levels of VEGF and its receptors have been shown to be reduced. ${ }^{25-27}$ In recent years, VEGF gene-modified EPCs have been successfully transplanted into patients with ischemic heart diseases or limb ischemia diseases to facilitate revascularisation, repair injured tissues and improve cardiac function. ${ }^{18}$

In this study, we found that 21 days after EPC transplantation, the mean basal ICP value of the trial group was remarkably higher than those of the blank-plasmid and control groups $(P<0.01)$. VEGF165 gene-modified EPCs, therefore, significantly enhanced resting blood flow in the corpus cavernosa of rats with diabetic ED. Additionally, after electrical stimulation, the mean ICP of the trial group remained remarkably higher than those of the blank-plasmid and control groups $(P<0.01)$, indicating that the intracavernosal injection of EPCs transfected with the VEGF165 gene restored the erectile function of rats with diabetic ED.
In this paper, we found that the VEGF165 expression level of EPCs transfected with a recombinant plasmid containing a VEGF165 expression cassette was significantly higher than that of EPCs transfected with a blank-plasmid or untransfected EPCs. This indicated that while EPCs themselves secrete limited amounts of VEGF165, transfection with the VEGF165 gene was able to enhance VEGF165 secretion of EPCs $(P<0.05)$. A total of 21 days after EPC transplantation, serum levels of the VEGF165 protein were also found to be remarkably enhanced. In this paper, we assayed EPCs in culture and in the corpora cavernosa 3 weeks after EPC transplantation and observed enhanced expression of VEGF165 both in vitro and in vivo, which was consistent with the results of the ELISA assay.

Twenty-one days after EPC transplantation, fluorescence microscopy showed that the EPCs had proliferated and incorporated into the new endangium of the corpora cavernosa of the rats. This phenomenon was especially notable for EPCs that had been transfected 
with pcDNA3.1(+)/VEGF165 prior to transplantation. Previous studies have demonstrated that during the process of neovascularisation of ischemic tissues, EPCs bind to the walls of new vessels, proliferate and differentiate into vascular ECs that participate in angiopoiesis. ${ }^{21}$ Some studies have shown that intracavernosal injection of VEGF into diabetic ED rat models is greatly protective for ECs. Because VEGF facilitates mitosis, it can induce endothelial hyperplasia and hypertrophy to resist the EC apoptosis that often occurs in ED. ${ }^{28}$ Our study confirmed this finding in a rat model of diabetic ED. In addition, we believe that the differences in the number of cavernosal EPCs that were present between the trial group and other groups reflects the role of VEGF165 in inducing EPCs to proliferate and incorporate into the new endangium. At 21 days after EPC transplantation, the vascular density of the corpora cavernosa of the rats differed between the study groups; the vascular density of the trial group was higher than those of the other two groups, indicating that VEGF165 gene-modified EPCs could induce neovascularisation of the corpora cavernosa in rats with diabetic ED. There is evidence suggesting that both the VEGF gene and EPCs play a role in facilitating the neovascularisation of the corpora cavernosa of rats with diabetic ED. ${ }^{29}$ However, our study indicated that VEGF and EPCs acted synergistically and had a stronger facilitatory effect on neovascularisation than either entity did alone, resulting in improved blood circulation in the corpora cavernosa.

Intracavernosal injection of EPCs transfected with the VEGF165 gene can restore the erectile function of rats with diabetic ED. The mechanism for this improvement is as follows: (i) EPCs transfected with the VEGF165 gene enhance the expression of the VEGF165 protein; (ii) the synergy between VEGF165 and EPCs facilitates the process of neovascularisation; and (iii) EPCs incorporate into the endangium to improve the function of ECs. However, more studies should be performed regarding whether EPCs derived from rats with diabetic ED have the same function as those from healthy rats.

\section{ACKNOWLEDGMENTS}

This work was supported by the Natural Science Foundation of Chongqing, China (No. 2007BB5288).

\section{AUTHOR CONTRIBUTIONS}

XG and WYH developed the concept, designed the study, analyzed the data and drafted the manuscript. WYH and MQ performed the experiment. MZX participated in the design, statistical analysis and preparation of the final manuscript. MW, YZD, CDL, ZBT, JL and YC participated in implementation of the experiment, data acquisition and preparation of the final manuscript. XG, WYH and MZX obtained funding.

\section{COMPETING FINANCIAL INTERESTS}

The authors declare no competing financial interests.

1 de Berardis G, Franciosi M, Belfiglio M, di Nardo B, Greenfield S et al. Erectile dysfunction and quality of life in type 2 diabetic patients: a serious problem too often overlooked. Diabetes Care 2002; 25: 284-91.

2 Burke JP, Jacobson DJ, McGree ME, Nehra A, Roberts RO et al. Diabetes and sexual dysfunction: results from the Olmsted County study of urinary symptoms and health status among men. J Urol 2007; 177: 1438-42.

3 Byrne RR, Henry GD, Rao DS, Huynh TT, Pippen AM et al. Vascular endothelial growth factor restores corporeal smooth muscle function in vitro. J Urol 2001; 165: 1310-5.
4 Lee MC, El-Sakka Al, Graziottin TM, Ho HC, Lin CS et al. The effect of vascular endothelial growth factor on a rat model of traumatic arteriogenic erectile dysfunction. J Urol 2002; 167: 761-7.

5 Bivalacqua TJ, Deng W, Kendirci M, Usta MF, Robinson C et al. Mesenchymal stem cells alone or ex vivogene modified with endothelial nitric oxide synthase reverse ageassociated erectile dysfunction. Am J Physiol Heart Circ Physiol 2007; 292: H127890.

6 Sharpe EE 3rd, Teleron AA, Li B, Price J, Sands MS et al. The origin and in vivo significance of murine and human culture-expanded endothelial progenitor cells. Am J Pathol 2006; 168: 1710-21.

7 Timmermans F, Plum J, Yoder MC, Ingram DA, Vandekerckhove B et al. Endothelial progenitor cells: identity defined? J Cell Mol Med 2009; 13: 87-102.

8 Zhang SJ, Zhang H, Hou M, Zheng Z, Zhou J et al. Is it possible to obtain "true endothelial progenitor cells" by in vitro culture of bone marrow mononuclear cells? Stem Cells Dev 2007; 16: 683-90.

9 Lin MH, Zhao S, Miao R, Jia N, Li J. Construction of eukaryotic expression vector for VEGF165gene and its expression in rat bladder smooth muscle cells. Nan Fang Yi Ke Da Xue Xue Bao 2007; 27: 654-6. Chinese.

10 Wang YM, Liu B, Sun LC, Yan YD, Si Y et al. Construction of VEGF recombinant plasmid pcDNA/V and its expression in model rats with acute myocardial ischemia. Sheng Wu Gong Cheng Xue Bao 2006; 22: 220-5. Chinese.

11 Shen ZJ, Wang H, Lu YL, Zhou XL, Chen SW et al. Gene transfer of vasoactive intestinal polypeptide into the penis improves erectile response in the diabetic rat. BJU Int 2005; 95: 890-4.

12 Heaton JP, Varrin SJ, Morales A. The characterization of a bio-assay of erectile function in a rat model. J Urol 1991; 145: 1099-102.

13 Melman A, Zhao W, Davies KP, Bakal R, Christ GJ. The successful long-term treatment of age related erectile dysfunction with hSlo cDNA in rats in vivo. J Urol 2003; 170: 285-90.

14 Rogers RS, Graziottin TM, Lin CS, Kan YW, Lue TF. Intracavernosal vascular endothelial growth factor (VEGF) injection and adeno-associated virus-mediated VEGF gene therapy prevent and reverse venogenic erectile dysfunction in rats. Int $J$ Impot Res 2003; 15: 26-37.

15 Chancellor MB, Tirney S, Mattes CE, Tzeng E, Birder LA et al. Nitric oxide synthase gene transfer for erectile dysfunction in a rat model. BJU Int 2003; 91: 691-6.

16 Bivalacqua TJ, Usta MF, Champion HC, Adams D, Namara DB et al. Gene transfer of endothelial nitric oxide synthase partially restores nitric oxide synthesis and erectile function in streptozotocin diabetic rats. J Urol 2003; 169: 1911-7.

17 Yamanaka M, Shirai M, Shiina H, Tanaka Y, Tsujimura A et al. Diabetes induced erectile dysfunction and apoptosis in penile crura are recovered by insulin treatment in rats. J Urol 2003; 170: 291-7.

18 Devanesan AJ, Laughlan KA, Girn HR, Homer-Vanniasinkam S. Endothelial progenitor cells as a therapeutic option in peripheral arterial disease. Eur J Vasc Endovasc Surg 2009; 38: 475-81.

19 Rodriguez-Losada N, Garcia-Pinilla JM, Jimenez-Navarro MF, Gonzalez FJ. Endothelial progenitor cells in cell-based therapy for cardiovascular disease. Cell Mol Biol (Noisy-le-grand) 2008; 54: 11-23.

20 Matsuo Y, Imanishi T, Hayashi Y, Tomobuchi Y, Kubo T et al. The effect of endothelial progenitor cells on the development of collateral formation in patients with coronary artery disease. Intern Med 2008; 47: 127-34.

21 Kawamoto A, Gwon HC, Iwaguro H, Yamaguchi JI, Uchida S et al. Therapeutic potential of ex vivo expanded endothelial progenitor cells for myocardial ischemia. Circulation 2001; 103: 634-7.

22 Foresta C, Caretta N, Lana A, Cabrelle A, Palu G et al. Circulating endothelial progenitor cells in subjects with erectile dysfunction. Int J Impot Res 2005; 17: 288-90.

23 Wu JC, Chen IY, Wang Y, Tseng JR, Chhabra A et al. Molecular imaging of the kinetics of vascular endothelial growth factor gene expression in ischemic myocardium. Circulation 2004; 110: 685-91.

24 Aiello LP. Wong JS. Role of vascular endothelial growth factor in diabetic vascular complications. Kidney Int Supp/ 2000; 77: S113-9.

25 de Young L, Bella A, Howard J, Brock G. Arteriogenic erectile dysfunction alters protein expression within the cavernosal tissue in an animal model. J Sex Med 2005; 2 : 199-206.

26 Xie D, Kontos CD, Donatucci CF, Annex BH. Cholesterol feeding reduces vascular endothelial growth factor signaling in rabbit corporal tissues. J Sex Med 2005; 2 : 634-40.

27 Ryu JK, Shin HY, Song SU, Oh SM, Piao S et al. Downregulation of angiogenic factors and their downstream target molecules affects the deterioration of erectile function in a rat model of hypercholesterolemia. Urology 2006; 67: 1329-34.

28 Lin CS, Ho HC, Chen KC, Lin G, Nunes L et al. Intracavernosal injection of vascular endothelial growth factor induces nitric oxide synthase isoforms. BJU Int 2002; 89: 955-60.

29 Tepper OM, Galiano RD, Kalka C, Gurtner GC. Endothelial progenitor cells: the promise of vascular stem cells for plastic surgery. Plast Reconstr Surg 2003; 111 : 846-54. 